\title{
Comorbidome and short-term prognosis in hospitalised COPD patients: the ESMI study
}

\section{To the Editor:}

Chronic obstructive pulmonary disease (COPD) patients have an increased prevalence of cardiovascular disease, cancer or psychiatric diseases, among many other comorbid conditions. This is explained in part by smoking history, along with genetic factors, ageing and the low grade inflammation characteristic of COPD $[1,2]$. Although comorbidities may be present at the time of the initial diagnosis, their frequency increases with COPD progression, and are particularly common in patients with more advanced disease, especially in those hospitalised with acute exacerbations $[3,4]$. These comorbidities complicate the management of COPD patients, lengthen hospital stay, cause physical functional dependency, increase number of readmissions, and are associated with decreased survival in the short, medium and long term [4-6].

Divo et al. [7] recently reported that fifteen of 79 comorbidities studied were related with an increased mortality during follow-up. Based on the BODE cohort, these authors developed the COPD specific CO-morbidity TEst (COTE), a new comorbidity index similar to the Charlson index, alongside a graphic representation of prevalence and impact in mortality of these comorbidities mimicking the solar system, called "the COPD comorbidome" that visually conveys the prevalence of the disease (size of the circles) and the risk of death (proximity to the centre) [7,8].

However, the BODE cohort included predominantly ambulatory patients with fewer comorbidities at the study inclusion, so that the representation of this comorbidome may be different in other populations, such as patients hospitalised for exacerbation of COPD in which the associated diseases are more prevalent and severe. Our main objective was to replicate the comorbidome in patients hospitalised for COPD exacerbation using data from the ESMI study [4].

In brief, ESMI is a longitudinal, observational, multicentre study conducted in 70 internal medicine services in Spain. Inclusion criteria were: 1) age 40 years and older, 2) admission for a COPD exacerbation, and 3) forced spirometry with a post-bronchodilator forced expiratory volume in $1 \mathrm{~s}$ (FEV1) $<80 \%$ of predicted and an FEV1 to forced vital capacity ratio $<0.70$. Patients with a previous diagnosis of asthma or bronchiectasis as predominant disease, inability to perform spirometry or admission for reasons other than an exacerbation of COPD were excluded.

Comorbidity data were collected using the Charlson index, a standard scale with 15 chronic diseases graded for severity of disease. Additionally, other disorders and conditions were identified using a specific questionnaire that included several relevant chronic diseases not included in the Charlson index, such as depression, arterial hypertension, anaemia, arrhythmias or dyslipidaemia, among others. Also data on dyspnoea (modified Medical Research Council (mMRC) scale), functional status (Katz) and previous hospitalisations for COPD or other causes were collected. Finally, vital status and cause of death up to 3 months after discharge were assessed by the same investigators who treated the patients during index exacerbation and were responsible for subsequent monitoring.

Qualitative variables were expressed as absolute values and percentages, while quantitative variables were summarised as mean \pm SD. For time-dependent variables we used the Cox logistic regression and KaplanMeier statistics. The study was approved by the clinical investigation ethics committee of the Hospital Mútua de Terrassa, Barcelona, Spain (EO/0922_0709). All patients participated voluntarily and signed written informed consent forms. The comorbidome was plotted according to the original design of Divo et al. [7], using the size of the circles to express the prevalence in percentage of different comorbidities and the risk of death (proximity to the centre) based on the value of the hazard ratio.

Overall, 606 patients were included. Mean \pm SD age of the studied population patients was $72.6 \pm 9.9$ years (range 41-94 years); 594 (89.9\%) were men, with a mean \pm SD post-bronchodilator FEV1 of $43 \pm 21.2 \%$. The average score on the Charlson index was $3.1 \pm 2$. The most commonly observed comorbidities in this index were heart failure (32.8\%), diabetes without complications (28.4\%), and ischaemic heart disease $(20.8 \%)$. Regarding comorbidities not included in the Charlson index, the most frequent were arterial hypertension (63.4\%), osteoporosis (16\%), abdominal obesity (29\%), dyslipidaemia (34\%), anaemia (19.3\%), and psychological disorders: depression $(15 \%)$ or anxiety $(18.3 \%)$. Comorbidities with a prevalence greater than $10 \%$ or related with mortality are detailed in table 1 . 


\begin{tabular}{lcccc} 
TABLE 1 Comorbidities and mortality: Cox regression analysis & & \\
Comorbidity & Prevalence \% & Hazard ratio & $\mathbf{9 5 \%} \mathbf{~ C l}$ & p-value \\
\hline Systemic hypertension & 63.4 & 0.9 & $0.44-2.28$ & $\mathrm{NS}$ \\
Diabetes mellitus & 35.8 & 1.9 & $0.87-4.17$ & $\mathrm{NS}$ \\
Dyslipidaemia & 33.8 & 1.16 & $0.48-2.78$ & $\mathrm{NS}$ \\
Heart failure & 32.8 & 2.31 & $1.05-5.1$ & $<0.01$ \\
Abdominal obesity & 29.4 & 1.1 & $0.44-2.35$ & $\mathrm{NS}$ \\
Arrhythmias & 24.9 & 2.8 & $1.28-6.15$ & $<0.001$ \\
Ischaemic heart disease & 20.8 & 1.29 & $1.04-1.61$ & $<0.01$ \\
Anaemia & 19.3 & 0.59 & $0.20-1.76$ & $\mathrm{NS}$ \\
Anxiety & 18.3 & 0.55 & $0.29-1.30$ & $\mathrm{NS}$ \\
Peripheral vascular disease & 16.8 & 3.83 & $1.71-8.57$ & $<0.002$ \\
Kidney disease & 16 & 3.91 & $1.75-8.73$ & $<0.005$ \\
Osteoporosis & 15.8 & 2.1 & $0.91-4.63$ & $\mathrm{NS}$ \\
Depression & 15 & 3.24 & $1.02-10.1$ & $<0.01$ \\
Obstructive sleep apnoea & 12.2 & 3.49 & $0.47-25.87$ & $\mathrm{NS}$ \\
Cerebrovascular disease & 11.7 & 3.44 & $1.49-7.99$ & $<0.006$ \\
Malignant solid tumour & 13.2 & 0.58 & $0.21-1.50$ & $\mathrm{NS}$ \\
Ulcer disease & 10.4 & 1.85 & $0.25-13.73$ & $\mathrm{NS}$ \\
Dementia & 3.6 & 5.17 & $1.76-15.28$ & $\mathrm{p}<0.001$ \\
\hline
\end{tabular}

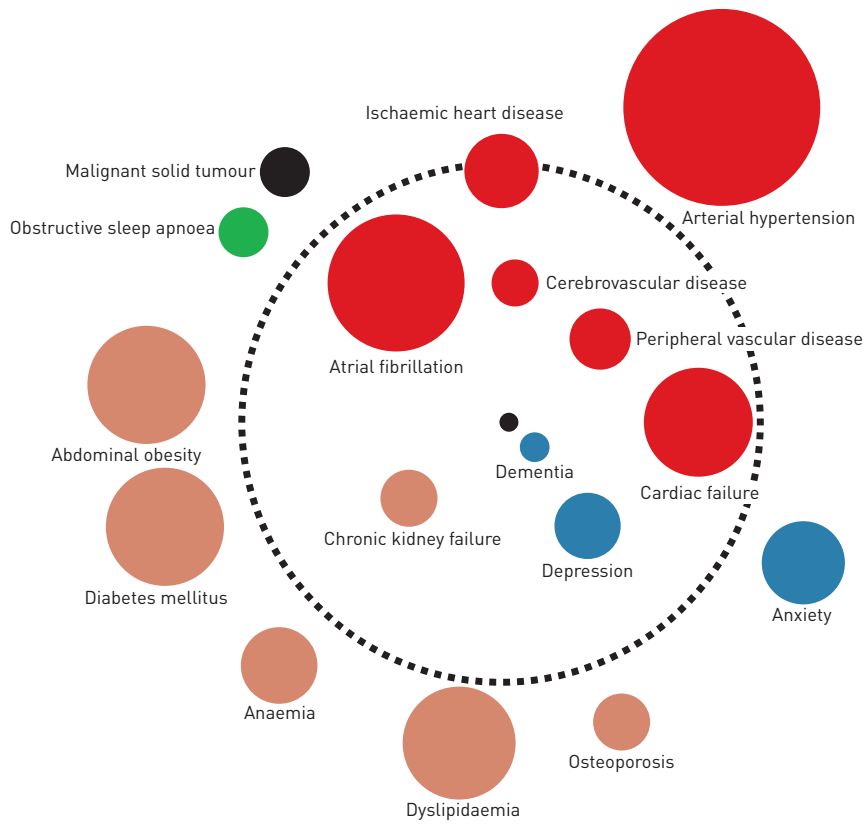

FIGURE 1 The area of the circle relates to the prevalence of the disease. The proximity to the centre (mortality) expresses the strength of the association between the disease and risk of death (hazard ratio). Bubble colours represent organ systems: 1) cardiovascular (red): arterial hypertension, ischaemic heart disease, atrial fibrillation, cardiac failure, peripheral artery disease, cerebrovascular disease; 2) metabolic disorders (brown): abdominal obesity, diabetes mellitus, dyslipidaemia, anaemia, chronic kidney failure, osteoporosis; 3) mental disorders (blue): depression, dementia, anxiety; 4) respiratory disease (green): obstructive sleep apnoea; 5) neoplasm (black).

27 patients $(4.5 \%)$ died up to 3 months after discharge. The causes of death were respiratory failure in 17 cases, stroke in three, cardiovascular in two, cancer in two, and other causes in three. Mortality at 3 months was associated with age, number of hospital admissions in the previous year, greater dyspnoea (mMRC), chronic home oxygen therapy, more functional dependence (Katz index), and comorbidities both measured with the Charlson index and total of comorbidities. Several individual comorbidities were also associated with 3-month mortality, such as ischaemic heart disease, heart failure, peripheral vascular disease, cerebrovascular disease, dementia, chronic kidney disease, depression or atrial fibrillation.

As shown in figure 1, the comorbidome in the ESMI study differs from that previously published by DIVO et al. [7], reflecting differences between the studied populations with different patterns and impact of 
comorbidities. Depression is not associated with mortality in the BODE cohort, although in the ESMI study its prevalence was high and significantly associated with mortality, similar to the findings of previous studies performed in hospitalised COPD patients [6]. Of note, the comorbidity with greater impact in mortality at 3 months was dementia, although its prevalence in our study was very low (3.6\%), probably reflecting a selection bias since all our patients met high quality spirometric criteria and it is well-known that many patients with dementia are not able to perform good quality spirometry [9-12].

The most frequent cause of death at 3 months in the ESMI study was respiratory failure, in spite of which several individual comorbidities are significantly related with mortality, reinforcing the importance of their combination in the individual patient prognosis. Indeed in many patients, COPD is just one more component of multiple comorbidities, and hence the term "multimorbidity" would be more accurate [13, 14].

A relevant difference and a probable limitation of our study compared with that previously published by Divo et al. [7] is the short follow-up time after discharge for COPD exacerbation ( 3 months). This is due mainly to the different objectives and thus differences in design between the two studies. The main purpose of the ESMI study was to explore prospectively comorbidities in COPD patients hospitalised for acute exacerbation in internal medicine services and to examine effects of them on short-term mortality. In contrast, the study of Divo et al. [7] was based on stable COPD outpatients, enrolled in pulmonary services with a lower comorbidity at inclusion and a longer follow-up, so the number and severity of comorbidities and their prognostic impact are clearly different. A second limitation is that inclusion and exclusion criteria in the ESMI study were necessarily restrictive in order to increase internal consistency so our results cannot be generalised to other populations.

In conclusion, our study reinforces the usefulness of a comorbidome as graphical representation of the prevalence and impact of comorbidities in patients hospitalised for COPD.

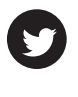

@ERSpublications

A comorbidome is a useful representation of the prevalence and impact of comorbidities in hospitalised COPD patients http://ow.ly/KYzSF

Pere Almagro $^{1}$, Francisco Javier Cabrera ${ }^{2}$, Jesus Diez-Manglano ${ }^{3}$, Ramon Boixeda ${ }^{4}$, Jesus Recio ${ }^{5}$, Joan Mercade ${ }^{1}$, Sergi Yun ${ }^{1}$ and Joan B. Soriano ${ }^{6}$ for the Working Group on COPD, Spanish Society of Internal Medicine

${ }^{1}$ Internal Medicine Service, Hospital Universitario Mútua de Terrassa, Barcelona, Spain. ${ }^{2}$ Internal Medicine Service, Hospital General Universitario Gregorio Marañón, Madrid, Spain. ${ }^{3}$ Internal Medicine Service, Hospital Royo Vilanova, Zaragoza, Spain. ${ }^{4}$ Internal Medicine Service, Hospital de Mataró, Barcelona, Spain. ${ }^{5}$ Internal Medicine Service, Hospital Universitario Vall d'Hebron, Barcelona, Spain. ${ }^{6}$ Instituto de Investigación Sanitaria Princesa (IP), Universidad Autónoma de Madrid, Madrid, Spain.

Correspondence: Pere Almagro, Hospital Universitario Mutua de Terrassa, Plaza Dr. Robert no. 5, Terrassa, Barcelona 08226, Spain. E-mail:19908pam@comb.cat

Received: Jan 172015 | Accepted after revision: March 172015 | First published online: April 212015

Support statement: The ESMI study was supported by Chiesi España. The sponsor had no role in the design of the study, the collection and analysis of the data, or the preparation of the manuscript.

Conflict of interest: Disclosures can be found alongside the online version of this article at erj.ersjournals.com

\section{References}

1 Vanfleteren LE, Spruit MA, Groenen M, et al. Clusters of comorbidities based on validated objective measurements and systemic inflammation in patients with chronic obstructive pulmonary disease. Am J Respir Crit Care Med 2013; 187: 728-735.

2 Faner R, Cruz T, López-Giraldo A, et al. Network medicine, multimorbidity and the lung in the elderly. Eur Respir J 2014; 44: 775-788.

3 Soriano JB, Visick GT, Muellerova H, et al. Patterns of comorbidities in newly diagnosed COPD and asthma in primary care. Chest 2005; 128: 2099-2107.

4 Almagro P, Cabrera FJ, Diez J, et al. Comorbidities and short-term prognosis in patients hospitalized for acute exacerbation of COPD. The ESMI study. Chest 2012; 142: 1126-1133.

5 Roberts CM, Stone RA, Lowe D, et al. Co-morbidities and 90-day outcomes in hospitalized COPD exacerbations. COPD 2011; 8: 354-361.

6 Almagro P, Calbo E, Ochoa de Echagüen A, et al. Mortality after hospitalization for COPD. Chest 2002; 121: 1441-1448.

7 Divo M, Cote C, de Torres JP, et al. Comorbidities and Risk of Mortality in Patients with COPD. Am J Respir Crit Care Med 2012; 186: 155-161.

8 Fabbri LM, Beghé B, Agustí A. COPD and the Solar System. Am J Respir Crit Care Med 2012; 186: 117-119.

9 Carvalhaes-Neto N, Lorino $\mathrm{H}$, Gallinari C, et al. Cognitive function and assessment of lung function in the elderly. Am J Respir Crit Care Med 1995; 152: 1611-1615.

10 Allen SC, Baxter M. A comparison of four tests of cognition as predictors of inability to perform spirometry in old age. Age Ageing 2009; 38: 537-541. 
11 Allen SC, Yeung P. Inability to draw intersecting pentagons as a predictor of unsatisfactory spirometry technique in elderly hospital inpatients. Age Ageing 2006; 35: 304-306.

12 Dodd JW, Charlton RA, van den Broek MD, et al. Cognitive dysfunction in patients hospitalized with acute exacerbation of COPD. Chest 2013; 144: 119-127.

13 Clini EM, Beghé B, Fabbri LM. Chronic obstructive pulmonary disease is just one component of the complex multimorbidities in patients with COPD. Am J Respir Crit Care Med 2013; 187: 668-671.

14 American Geriatrics Society Expert Panel on the Care of Older Adults with Multimorbidity. Guiding principles for the care of older adults with multimorbidity: an approach for clinicians. J Am Geriatr Soc 2012; 60: E1-E25.

\title{
Increased wheezing risk with diesel exposure among children of younger mothers
}

\author{
To the Editor:
}

Lower respiratory tract illnesses (LRIs) are the largest cause of mortality among young children worldwide [1], and are associated with altered lung function, immune system development and respiratory disease in adulthood [2,3]. Several studies have demonstrated associations between wheezing LRIs in early life and exposure to diesel traffic related air pollutants (D-TRAP) $[1,4,5]$. We, along with others, have previously demonstrated that younger maternal age at birth is also a risk factor for wheezing LRIs in the first year of life $[6,7]$. Why children of younger mothers are more susceptible is unknown, but both altered responses to environmental challenges in their offspring due to maternal immaturity [6] and increased socioeconomic and environmental risks have been proposed as causes [8].

We postulated that children of younger mothers are more susceptible to environmental exposures than children of older mothers. To test this hypothesis, we used data from the Tucson Children's Respiratory Study, a longitudinal birth cohort designed to investigate risk factors for LRIs in early childhood and chronic lung disorders later in life $[9,10]$. Through age 3 years, LRIs were diagnosed by study physicians who documented wheeze [10]. Parents were asked if their child had active wheeze during the past year at 6 years. Four wheezing phenotypes were defined: never, transient-early (only to age 3 years), late-onset (by age 6 but not by age 3 years), and persistent (by age 3 and 6 years) [11]. The study was approved by the University of Arizona Human Subjects Committee. Informed consent was obtained from a parent of a participating child.

We estimated D-TRAP exposure for each enrolment address using CAL3QHC, a Gaussian line-source dispersion model [12], that estimates traffic pollutant concentrations as a function of distance to roadway, traffic volume, wind speed and direction, atmospheric stability class, and vehicle emissions. We only used highways in our analysis, as they represent the primary source of diesel exhaust in Tucson, AZ, USA. Roadway and meteorological data corresponding to each participant's season of birth were obtained from the Arizona Department of Transportation and the National Weather Service. Emissions factors were computed with the United States Environmental Protection Agency's MOBILE 6.2 model for PM2.5 by diesel vehicle class.

We geocoded each participant's enrolment address using ArcGIS 10 (ESRI, Redlands, CA, USA), and obtained D-TRAP exposure for these coordinates with CAL3QHC. D-TRAP estimates represent seasonal exposures to a mixture of air pollutants from proximity to highway diesel traffic emissions independent of other diesel exhaust sources, and are interpreted as a unitless index.

Because our outcome was a count variable (i.e. number of wheezing LRIs) and $62 \%$ had no wheezing LRI (mean 0.62, variance 1.00), we used a negative binomial regression to account for over-dispersion in assessing the relationship between D-TRAP exposure and wheezing LRI events. Multinomial regression was used to assess associations with wheezing phenotypes. Interaction terms were included to assess effect modification. We examined pairwise associations between D-TRAP exposure, maternal age, and wheezing LRIs, with potential confounders (i.e. sex, ethnicity, maternal education, household cooling methods, older children in the home, marital status and census tract socioeconomic status, among others). We used the robust cluster estimator of variance to account for potential clustering by census tract. To assess multi-collinearity, we calculated the variance inflation factor for the final adjusted models. STATA 13.0 (StataCorp, College Station, TX, USA) was used for all analyses. 